\title{
Preparation and Characterization of Galanga Essential Oil Liposomes
}

\author{
Neng Liü, b, Tian Guan a , Wei Zhou ${ }^{\text {a, * }}$, Jihua Li ${ }^{\text {a }}$, Yupo Cao ${ }^{\text {a }}$, Lijing Lin ${ }^{\text {a }}$ \\ ${ }^{a}$ Agricultural Products Processing Research Institute, Chinese Academy of Tropical Agricultural \\ Sciences, Zhanjiang 524000, China \\ ${ }^{b}$ School of Food Science and Technology, Huazhong agricultural University, Wuhan 430000, China \\ *weizhou111@foxmail.com
}

Key words: Galanga essential oil liposomes, Ethanol injection method, Preliminary stability test, Characterization.

Abstract. Galanga essential oil (GEO) liposomes were prepared by ethanol injection method and were characterized by size, polydispersity index (PDI) and zeta potential. Furthermore, the quality evaluation was conducted using the morphology observation and preliminary stability test. It was shown that Galanga essential oil-loaded liposomes embraced a mean particle size of $61.87 \pm 0.21 \mathrm{~nm}$, the zeta potential was $-16 \pm 0.99 \mathrm{mV}$, respectively. According to electron microscope observation, GEO liposomes signified a spherical structure. Preliminary stability studies pointed out that it exhibited good stability in a light-avoiding environment at $4{ }^{\circ} \mathrm{C}$ with stable size distribution.

\section{Introduction}

Galanga is a member of the Zingiberaceae family which is a pungent and aromatic dry rhizome. A galanga is an essential spice and food flavoring product as well as a medicament or part of medicaments in Asian folk medicine for various applications, such as against rheumatic oilments, for the treatment of respiratory diseases, as aromaticum and tonicum, but also as aphrodisiacum [1]. It has various pharmacological activities including anticancer [2], anti-oxidant [3], antihypertensive [4], anti-inflammatory [5] and antibacterial [6]. Galangal essential oil is one of the secondary metabolites which exists in galangal rhizome and is an aromatic and volatile oily liquid [7]. Pharmacological study showed that the galangal essential oil has antimicrobial activity and antioxidant effect, but its maintenance phase of antimicrobial activity is pretty short due to the unstability and effumability.

Bilayer vesicle structure self-assembled of phospholipid when present in an aqueous environment, as an excellent vector, are widely used in medicine, food and cosmetics. The double-layed structure of liposomes possesses special amphiphilic characteristics. Liposomes are spherical colloidal particles in which the internal aqueous cavity is surrounded by a self-assembled lipid membrane. Liposomes have many excellent properties, such as biocompatibility, targeting and slow-releasing potential [8]. They represent an efficient approach for incorporating natural compounds, such as essential oil components, by improving their solubility and chemical stability [9]. There are so many studies on the use of liposome technology to encapsulate the essential oil [10-12].

In this paper we focus on determining the characteristics of liposomes after encapsulating Galanga Essential Oil, prepared by the ethanol injection method. First, the effects of Galanga Essential Oil components on physicochemical properties of liposomes were studied by measuring the mean particle size, zeta potential and polydispersity index. Second, the morphology properties of liposomes systems were analyzed by atomic force microscope (AFM) and transmission electron microscope (TEM). Finally, a storage stability of the liposomes was studied at $4{ }^{\circ} \mathrm{C}$ with size distribution, so as to provide science foundation for the quality control of GEO Liposomes. It is of great practical significance to prepare GEO liposomes, which offers theoretical instruction for regulating effectively antibacterial activities in natural food antiseptic.

\section{Materials and methods}

Materials.Galangal essential oil was provided by the agricultural products processing institute of the 
Chinese Academy of Tropical Agricultural Sciences (Zhanjiang, China). Phospholipid S75 was purchased from Lipoid GmbH (Germany). Cholesterol (WXBB1238) was obtained from Sigma-Aldrich Chemical Co. (USA). Ethanol, Tween 80, Vitamin E and other materials were all of analytical grade. Phosphate buffer (pH7.4).

\section{Methods}

\section{Preparation of GEO liposomes}

The mass ratio of galangal essential oil, VE, tween 80, phospholipid and cholesterol was 10: 1.2: 15: 60: 8. They were dissolved in sufficient dehydrated alcohol with a magnetic stirrer (IKARCT, Guangzhou, China). The obtained ethanol solution was injected slowly into phosphate-buffered saline ( $\mathrm{pH} 7.4$, $0.05 \mathrm{M}$ ) using a syringe pump and stirred vigorously for 30 minutes. The obtained suspension was transferred to an appropriate round bottom flask and submitted to a rotary evaporator (RE-2000, Yarong Biochemistry Installation Factory, Shanghai, China) at a proper temperature to evaporate the dehydrated alcohol. The final concentration of galangal essential oil is $1 \mathrm{mg} / \mathrm{mL}$. GEO nanoliposomes was obtained by the super-high pressure viscolizer (micro-jet, Langfang general machine Ltd, Langfang, China) at a pressure of $120 \mathrm{MPa}$ [13].

\section{Characterization of GEO liposomes}

Mean particle size and polydispersity index measurements

The appropriate GEO liposomes samples were diluted 10-fold with distilled water and stirred using a vortex mixing oscillation apparatus (Guangzhou, China) before determination. Size and PDI of Samples were measured by the laser particle size analyser (Nicomp 380/ZLS, Pss Corporation, USA) at $25^{\circ} \mathrm{C}$. Samples were scattered at $635 \mathrm{~nm}$ (angle of 90 degrees). Each sample was measured in triplicate.

\section{Zeta potential analysis}

By setting refraction (1.333), viscosity (0.933) and laser wavelength (635 nm), zeta potential values were measured using a laser particle size analyser at $25^{\circ} \mathrm{C}$. All determinations were carried out in triplicate.

\section{The morphology of GEO liposomes}

The morphology and structure of GEO liposomes were observed by TEM (JEM-2100, Jeol Corporation, Japan) and AFM (Agilent 5500, USA). Diluted 1000 times by ultrapure water, the GEO liposomes sample was applied to mica plate. After air-dried at room temperature, the sample was examined by AFM in tapping mode. The diluted sample was added to a carbon-coated copper grid and negatively stained with $1 \%$ uranium acetate for 2 minutes. The excess liquid was removed by filter paper. Observation was conducted by TEM at an accelerating voltage of $120 \mathrm{kV}$.

\section{Preliminary stability study}

GEO liposomal suspensions prepared by the ethanol injection method were stored in a capped-glass container under low temperature $\left(4^{\circ} \mathrm{C}\right)$ and dark condition. The samples were observed at specified intervals (days $0,5,15,45,60$ ). The physical stability of vesicle dispersions was evaluated in terms of changes in the mean particle size and polydispersity index.

\section{Results and discussion}

Size, Polydispersity index and Zeta potential

Table 1 The size, PDI and zeta potential value of GEO liposomes

\begin{tabular}{ccc}
\hline average diameter $(\mathrm{nm})$ & PDI & Zeta potential $(\mathrm{mV})$ \\
\hline $61.87 \pm 0.21$ & $0.32 \pm 0.008$ & $-16 \pm 0.99$ \\
\hline
\end{tabular}




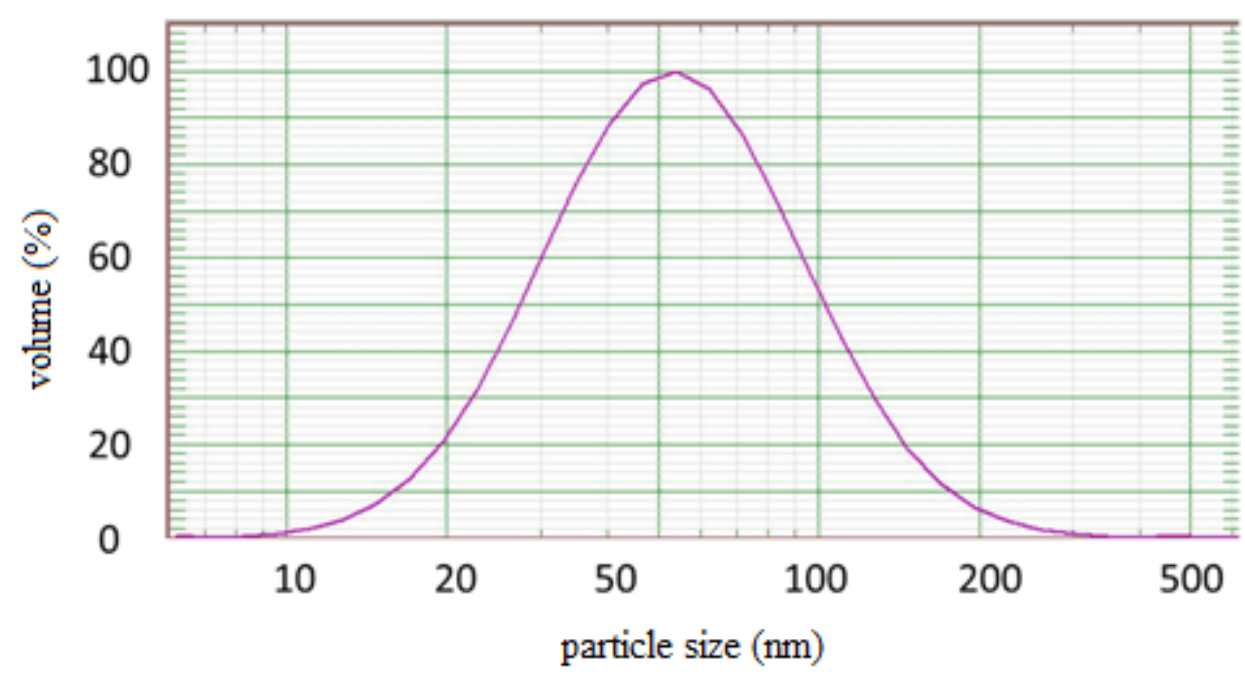

Fig. 1 The size distribution of GEO liposomes

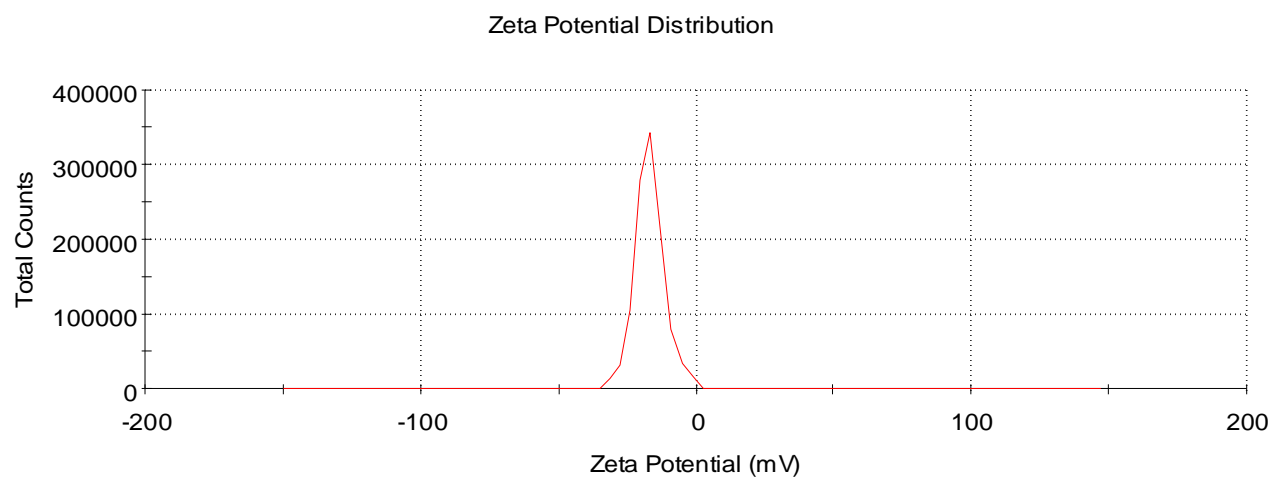

Fig. 2 The zeta potential distribution of GEO liposomes

A roughly normal distribution, with a mean of $61.87 \pm 0.21 \mathrm{~nm}$, was observed in particle size of GEO liposomes (Fig. 1). The more the particle diameter distribution is narrower, the more the particles size is more uniform. It is related to the storage stability and transdermal absorption of liposomes [14]. Furthermore, the PDI values were between 0.312 and 0.328 , indicating a good homogeneous population (Table 1).

Zeta potential is an important and useful indicator of particle surface charge, which can be used to predict and control the stability of colloidal suspensions or emulsions [15]. From Fig. 2, GEO liposomes were possessed of negative charge and their zeta potential were $-16 \pm 0.99 \mathrm{mV}$. Experimentally, the negative liposomes were stable, and the positive liposomes were unstable [16]. In addition, it is currently admitted that zeta potentials under $|30| \mathrm{mV}$, optimum $>|60| \mathrm{mV}$, are required for full electrostatic stabilization; potentials between $|5|$ and $|15| \mathrm{mV}$ are in the region of limited flocculation [17]. However, this rule cannot strictly be applied for systems which contain steric stabilizers, because the adsorption of steric stabilizers will decrease the zeta potential due to the shift in the shear plane of the particle [15]. GEO liposomal systems contain steric stabilizer (Tween 80), which results in an unreasonably low measuring result (Fig. 2). The same phenomenon was also observed by Schwarz et al. [18] with solid lipid structures. 


\section{Morphological observation}

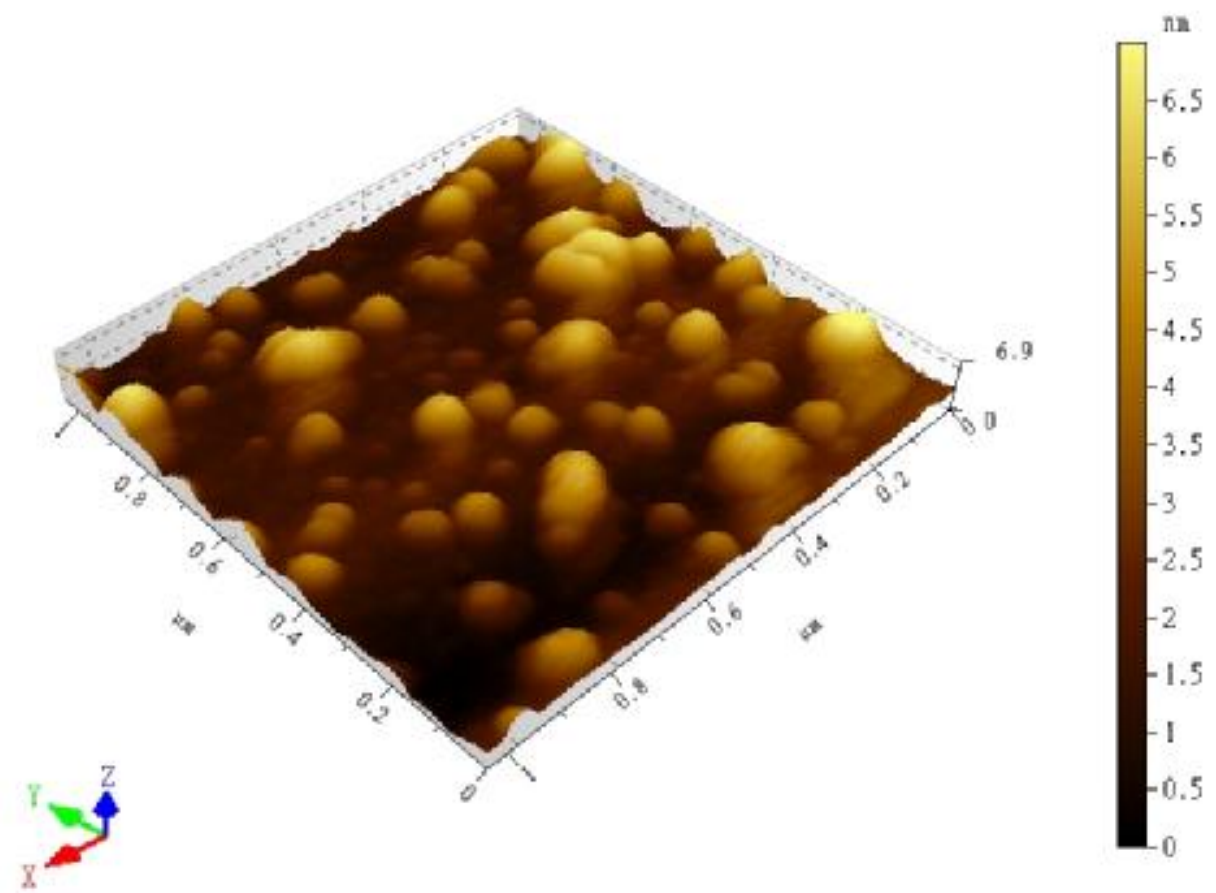

Fig. 3 The 3D atomic force microscopic images of GEO liposomes

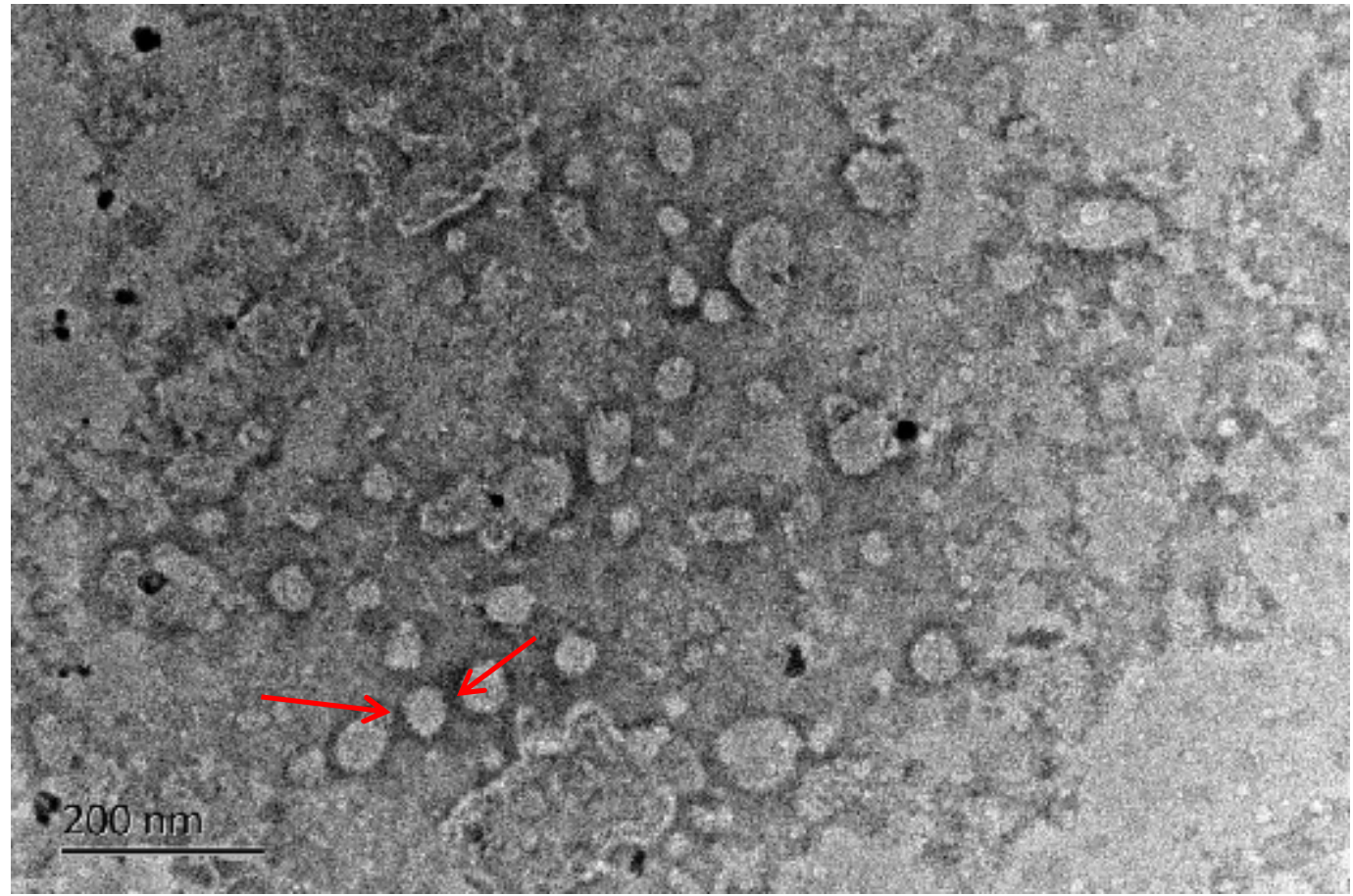

Fig. 4 Transmission electron micrographs of GEO liposomes

The 3D profile and surface morphology of GEO liposomes were analyzed using AFM. Many spherical or ellipsoidal particles were observed in Fig. 3. In addition, the majority of particle diameter is the main range of around $60 \mathrm{~nm}$ (Fig. 3). The results were also consistent with previous conclusion (Fig. 1, Table 1). The TEM images of GEO liposomes showed the formation of nanometer-sized of spherical or ellipsoidal shape (Fig. 4), indicating particles size distribution is comparatively uniform. Thus we can see that galangal essential oil encapsulated into liposomes using the ethanol injection method had a narrow size distribution. 
However, some non-spherical or large particles were observed in Fig. 3 and Fig. 4, respectively. This is probably because of galangal essential oil changed or weakened the structure of liposomes. Research founded that the accumulation of lipophilic compounds in the hydrophobic part of the membrane will disturb the interactions between the acyl chains of the phospholipids, leading to the swelling of the bilayer [19]. Valenti et al. [20] founded the size of liposomes (MLV and SUV) encapsulating Santolina insularis essential oil showed a slight increase after 180 days. Carine Sebaaly et al. [8] studied the TEM images of Eug-or clove essential oil-loaded S100-liposomes. Non-spherical structures were also observed in some images.

Preliminary Stability study of GEO liposomes

Table 2 Effects of storage time on the size distribution and PDI of GEO liposomes

\begin{tabular}{cccccc}
\hline Time $(\mathrm{d})$ & 0 & 5 & 15 & 45 & 60 \\
\hline Size $(\mathrm{nm})$ & $61.87 \pm 0.21$ & $63.93 \pm 0.42$ & $63.7 \pm 0.14$ & $57.67 \pm 0.25$ & $62.27 \pm 0.25$ \\
PDI & $0.32 \pm 0.008$ & $0.29 \pm 0.009$ & $0.29 \pm 0.009$ & $0.3 \pm 0.007$ & $0.30 \pm 0.003$ \\
\hline
\end{tabular}

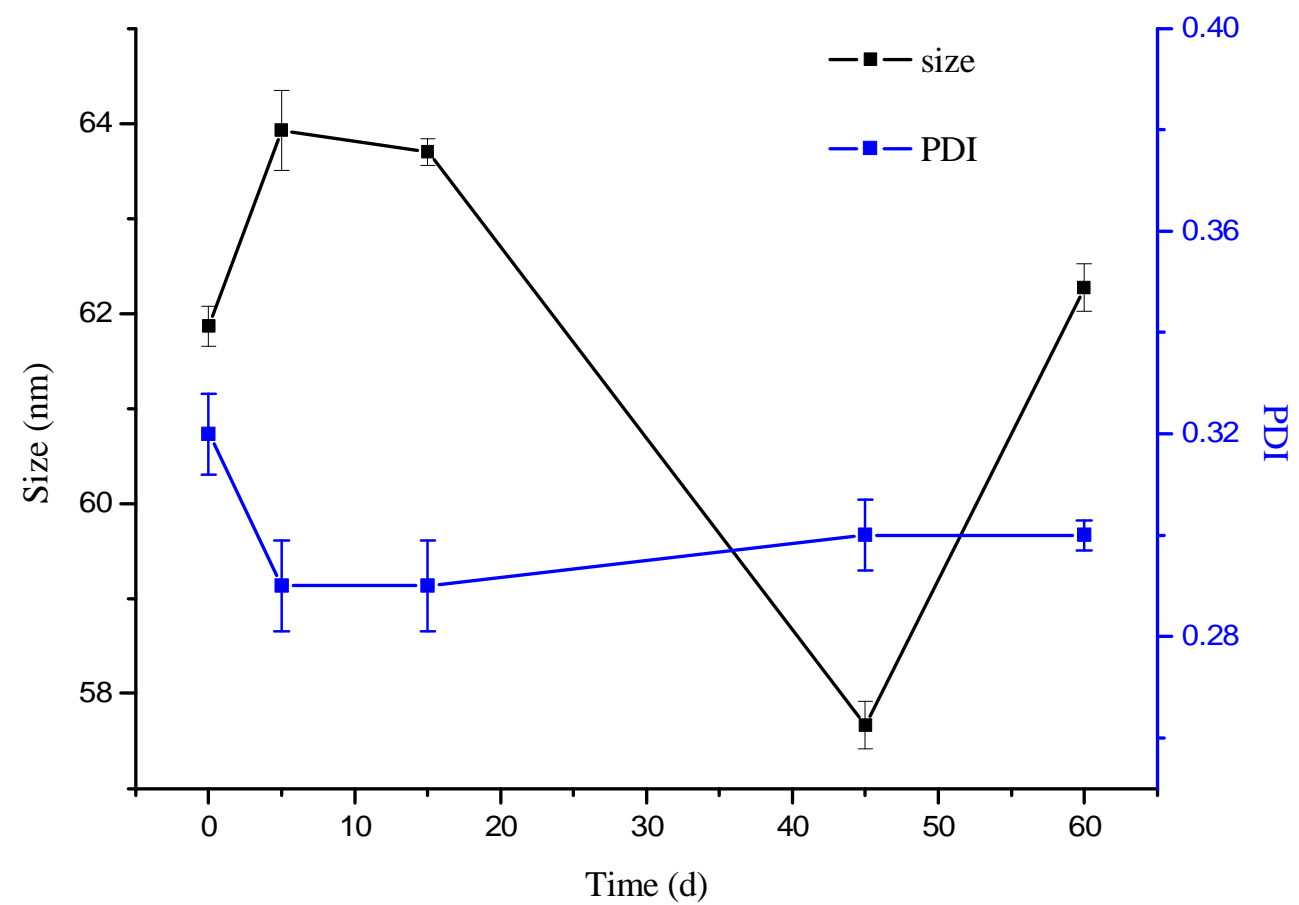

Fig. 5 Effects of storage time on stability of GEO liposomes 

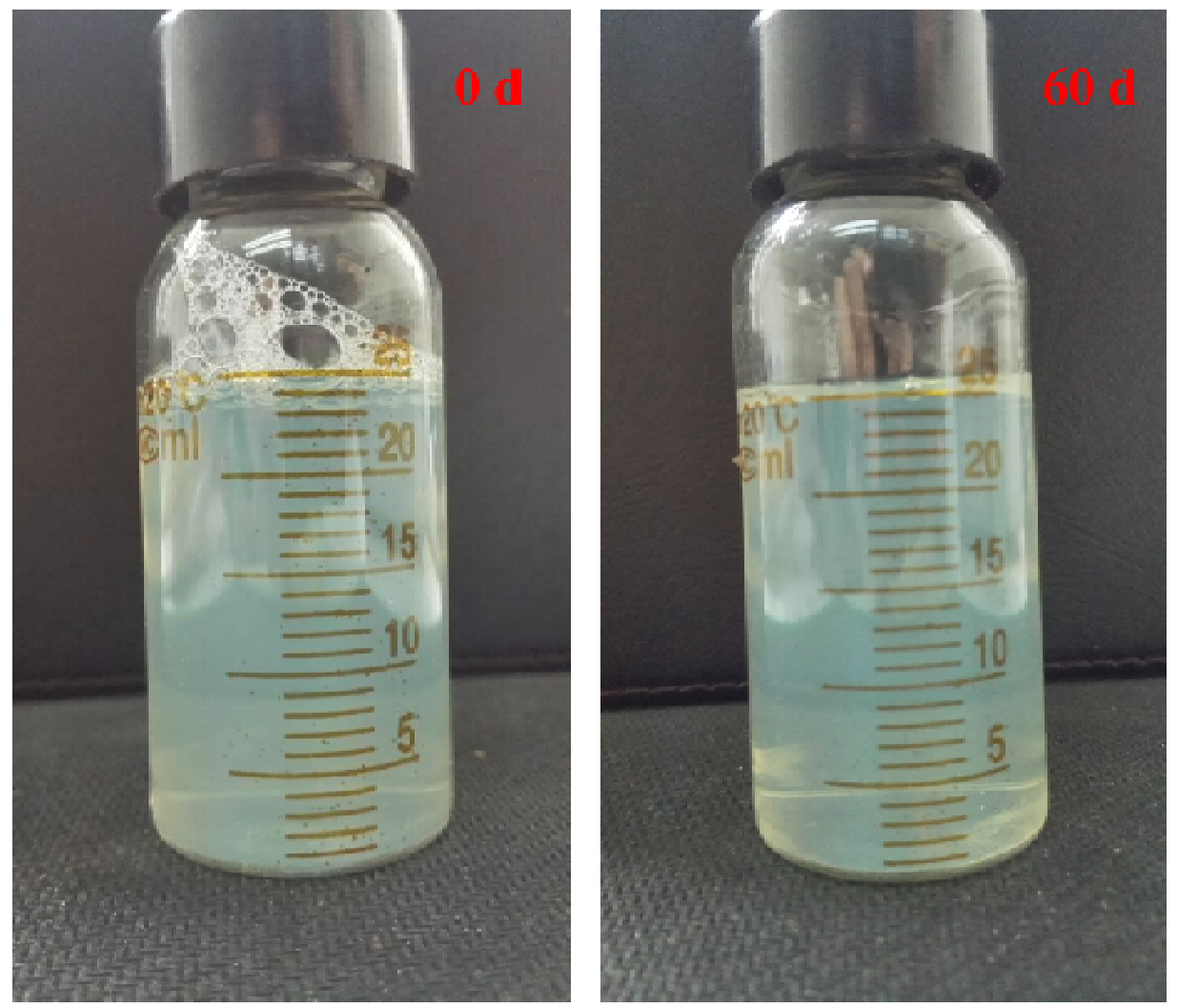

Fig. 6 Appearance of GEO liposomes under $4^{\circ} \mathrm{C}$ for 60 days

The mean particle size and PDI is an ideal marker to characterize the quality of GEO liposomes when they were stored at $4^{\circ} \mathrm{C}$, the results shown in Table 2 and Fig. 5, respectively. As shown in Table 2, a slightly increase of GEO liposomes size from $61.87 \pm 0.21$ to $63.93 \pm 0.42 \mathrm{~nm}$ were observed after stored for 5 days. The particle size did not change dramatically from storage days 5 to 15 . When GEO liposomes were stored for 45 days, the particle size declined to $57.67 \pm 0.25 \mathrm{~nm}$. At day 60 , the size of the liposomes showed an increase when compared to day 45. It can be seen intuitively from Fig. 5 that the PDI curve is relatively flat. And the PDI values, which ranged between 0.29 and 0.3 , remain unchanged. On the whole the size of GEO liposomes has minor change and the liposomal solution is homogeneous and stable perfectly. Similarly, from the Fig. 6, the appearance of GEO liposomal suspension did not appreciably change after 60 days of storage at $4^{\circ} \mathrm{C}$. This phenomenon also showed that the suspension remained a fine stability in this condition.

Similar findings also have been reported by other researchers. For example, Salima Varonaf [21] founded that Lavandin Essential Oil-loaded Liposomes showed a good stability for at least 1 month at $5^{\circ} \mathrm{C}$. The mean size of liposomes changed during the first 10 days and remained stable after this period, confirming that fusion and breakage of vesicles did not occur after the initial period of 10 days. Carine Sebaaly et al. [8] reported the PDI values of clove essential oil-loaded Phospholipon 80H-liposomes ranged between 0.22 and 0.33 after 2 months of storage at $4^{\circ} \mathrm{C}$, indicating a homogeneous size distribution. And the content of clove essential oil, measured after 2 months of storage at $4^{\circ} \mathrm{C}$, was consistent with those obtained after storing for 0 months at $4^{\circ} \mathrm{C}$. As reported by Chiara Sinico et al. [22], Artemisia arborescens L. essential oil-loaded liposomes were very stable for the first six months. Artemisia arborescens L. essential oil was very low and at least $95 \%$ of the essential oil was already incorporated into liposomes while particle sizes increased slightly (5-10\%).

\section{Conclusion}

In this paper, GEO liposomes were prepared using the ethanol injection method. The particle size, zeta potential and polydispersity index of the GEO liposomes were characterized respectively. The 
liposomal particles displayed the shape of spherical or ellipsoidal by AFM and TEM exposure. They were highly stable during their storage at $4^{\circ} \mathrm{C}$ under dark condition.

\section{Acknowledgements}

This research was supported by the National Natural Science Foundation of Hainan province (2015305) and the Science and Technology Plan Projects of Zhanjiang (2014C01005).

\section{References}

[1] L Jirovetz, G Buchbauer and M P Shafi: submitted to Acta Pharmaceutica (2003)

[2] S K Bagya, P V Rajashree and K G Sam: submitted to Research Journal of Medicinal Plant (2011)

[3] N Rao, D Kaladhar: submitted to World J of Pharmacy and Pharmaceutical Sci (2014)

[4] R H Othman, M A Mohd and K Awang: submitted to Planta Medica (2002)

[5] A M Vittalrao, T Shanbhag and M Kumari: submitted to Indian J Physiol Pharmacol (2011)

[6] A Hamad, A Alifah and A Permadi: submitted to International Food Research Journal (2016)

[7] S A Khatibi, A Misaghi and M H Moosavy: submitted to Pharmaceutical Sciences (2015)

[8] C Sebaaly, A Jraij and H Fessi: submitted to Journal of Power Sources (2003)

[9] C Maria, I Benedetta and V B Louis: submitted to International Journal of Pharmaceutics (2011)

[10] C B Detoni, D M de Oliveira and I E Santo: submitted to Journal of liposome research (2012).

[11] P A Yoshida, D Yokota and M A Foglio: submitted to Journal of microencapsulation (2010)

[12] E Moghimipour, N Aghel and M A Zarei: submitted to Industrial \& Engineering Chemistry Research (2012)

[13] S Peng, L Zou and W Liu: submitted to Journal of Food Protection (2015)

[14] K A Edwards, A J Baeumner: submitted to Talanta (2006)

[15] B Heurtault, P Saulnier and B Pech: submitted to Biomaterials (2003)

[16] D J A Crommelin: submitted to Journal of pharmaceutical sciences (1984)

[17] P Ney: Zeta-Potentiale und Flotierbarkeit von Mineralen (Springer, New York 1973)

[18] C Schwarz, W Mehnert and ller: submitted to Journal of Controlled Release (1994)

[19] J Sikkema, J A D Bont and B Poolman: submitted to Microbiological Reviews (1995)

[20] D Valenti, A De Logu and G Loy: submitted to Journal of Liposome Research (2001)

[21] S Varona, Ángel Martín and M J Cocero: submitted to Industrial \& Engineering Chemistry Research (2011)

[22] C Sinico, A De Logu and F Lai: submitted to European Journal of Pharmaceutics and Biopharmaceutics (2005) 